\title{
Monoarthritis of the Elbow Revealing Osteoid Osteoma: A Case Report and Literature Review
}

\author{
Najlae El Ouardi ${ }^{1 *}$ (D), Siham Sadni ${ }^{1}$, Laila Taoubane ${ }^{1}$, Aziza Mounach ${ }^{1}$, Ahmed Bezza ${ }^{1}$
}

\begin{abstract}
Osteoid osteoma is a rare benign osteoblastic tumor that mainly occurs in the extra-articular segments of lower limb long bones. The elbow is an exceptional location for osteoid osteoma.

The diagnosis is usually delayed since the elbow is a rare site for this tumor and unspecific clinical signs, such monoarthritis, may mimic other more common conditions.

We report the case of a 16-year-old girl who presented with chronic monoarthritis of the elbow which was treated falsely as tuberculous arthritis and monoarticular rheumatoid involvement.

The laboratory tests were negative. Computed tomography scan and magnetic resonance imaging showed the focal nidus at the coronoid process of the ulna with mild synovitis. The diagnosis of osteoid osteoma was not made until the disease had been progressing for eighteen months and the elbow had already become stiff.

Arthroscopic excision of the lesion was performed, and histopathology of the nidus was consistent with osteoid osteoma. Since then, the patient has been pain-free with an improved elbow range of motion.

\section{Keywords}

Osteoid Osteoma; Elbow; MonoArthritis; Nidus

${ }^{1}$ Rheumatology Department, Mohammed V Military Academic Hospital, Faculty of Medicine and Pharmacy, Mohammed V-Souissi University, Rabat, Morocco
\end{abstract}

*Corresponding author: doc.najlae89@gmail.com

\section{Introduction}

Osteoid osteoma is a painful benign osteoblastic tumor that most commonly affects young patients. It mainly involves the diaphysis of lower limb long bones (the femur and the tibia in $70 \%$ of cases) $[1,2]$. Elbow localization is very rare, accounting for about $3 \%$ of all osteoid osteoma [1].

Diagnosing osteoid osteoma of the elbow is challenging since the elbow is an extremely rare site of osteoid osteoma and clinical signs are not specific [3]. If misdiagnosed and mistreated, it can occasionally progress to elbow stiffness due to prolonged inflammation and synovitis $[4,5]$.

We report the case of a 16-year-old girl with chronic monoarthritis and stiffness of the elbow as a result of osteoid osteoma that was falsely treated as tuberculous arthritis and monoarticular rheumatoid involvement.

\section{Case Presentation}

A 16-year-old girl was admitted to our hospital due to chronic monoarthritis and stiffness of the left elbow. Elbow pain started suddenly eighteen months ago. It was persistent but exacerbated at night and partially improved with the use of non-steroidal anti-inflammatory drugs (NSAIDs).

The patient had no other musculoskeletal complaints, no history of trauma or a significant past medical history.

The symptoms were previously attributed to tuberculous arthritis of the elbow and the patient received a ninemonth chemotherapy that resulted in elbow stiffness. Afterwards, monoarticular rheumatoid involvement was considered as a potential diagnosis and the patient received methotrexate for 6 months; however, there was no relief.

On clinical examination of the left elbow, the joint was tender with localized swelling; no erythema or heat were noted. There was a restriction in both flexion and extension with elbow range of motion of $30^{\circ}$ to $100^{\circ}$. Pronation and supination were unrestricted.

There were no other osteoarticular abnormalities; the neurological and vascular examinations were normal.

Biological studies showed erythrocyte sedimentation rate (ESR) of $10 \mathrm{~mm} / 1 \mathrm{~h}, \mathrm{C}$-reactive protein $(\mathrm{CRP})<1 \mathrm{mg} / \mathrm{l}$, normal range of white blood cell count. Infectious and immunological tests were negative.

Left elbow X-rays showed sclerosis of the proximal ulnar aspect (Fig. 1). Computed tomography (CT) scan 


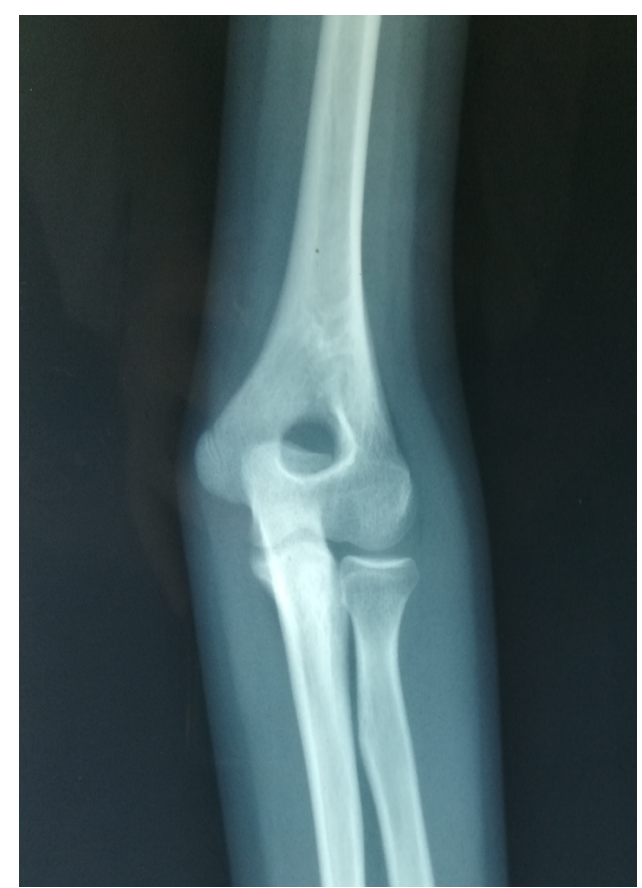

A

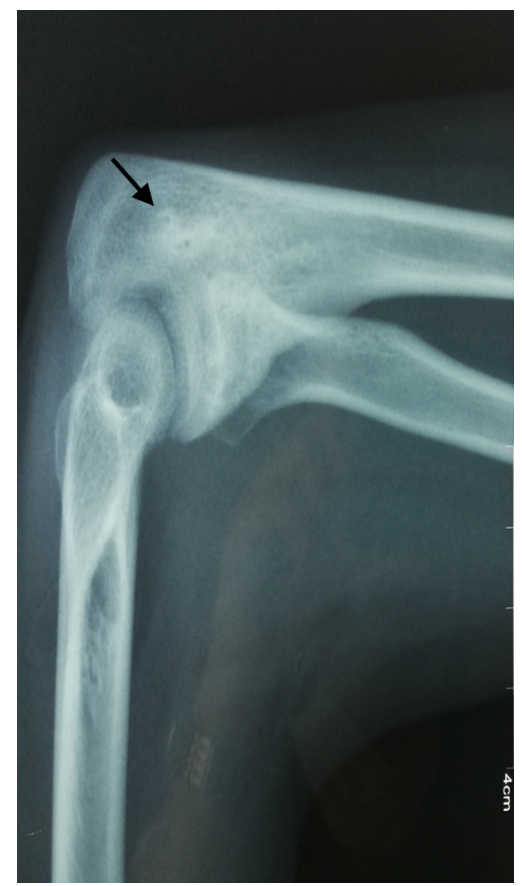

B

Figure 1. Plain radiograph images of the left elbow revealing sclerosis of the proximal ulnar aspect (arrow).

revealed a focal nidus at the coronoid process with marked sclerotization of the surrounding bone (Fig. 2).

The lesion measured $12.8 \mathrm{~mm} \times 11 \mathrm{~mm} \times 8 \mathrm{~mm}$. These findings were consistent with a diagnosis of osteoid osteoma. Magnetic resonance imaging (MRI) showed the nidus with signs of mild synovitis (Fig. 3).

Arthroscopic resection was performed: en bloc excision of the lesion was applied; the nidus and the circumferential sclerotic bone were curetted. Histopathology of the nidus was consistent with osteoid osteoma.

One month after surgery and physiotherapy, the patient

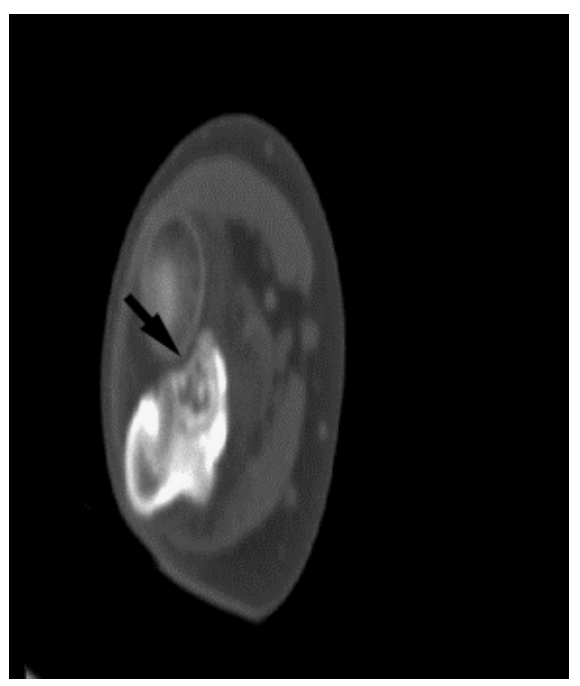

A reported complete pain relief and improvement of elbow flexion and extension with elbow range of motion of $50^{\circ}$ to $110^{\circ}$.

\section{Discussion}

Osteoid osteoma is a benign tumor that mainly occurs in the first three decades of life $(80 \%)$, with a predominance in men. It is usually embedded within the long bones of the lower limbs $(50 \%)$ [1, 6].

Elbow localization at the juxta-articular level is rare.

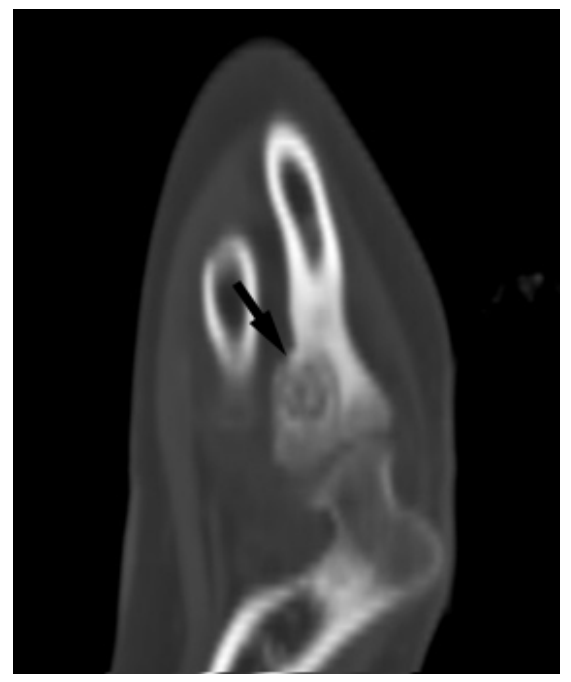

B

Figure 2. Coronal (A) and sagittal (B) computed tomography scans of the left elbow showing the focal nidus at the coronoid process with marked sclerotization of the surrounding bone (arrow). 


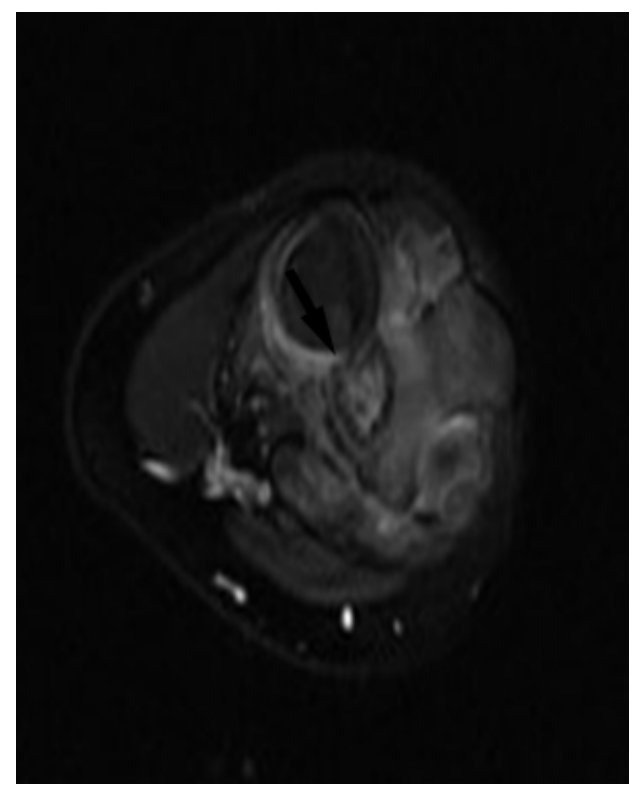

A

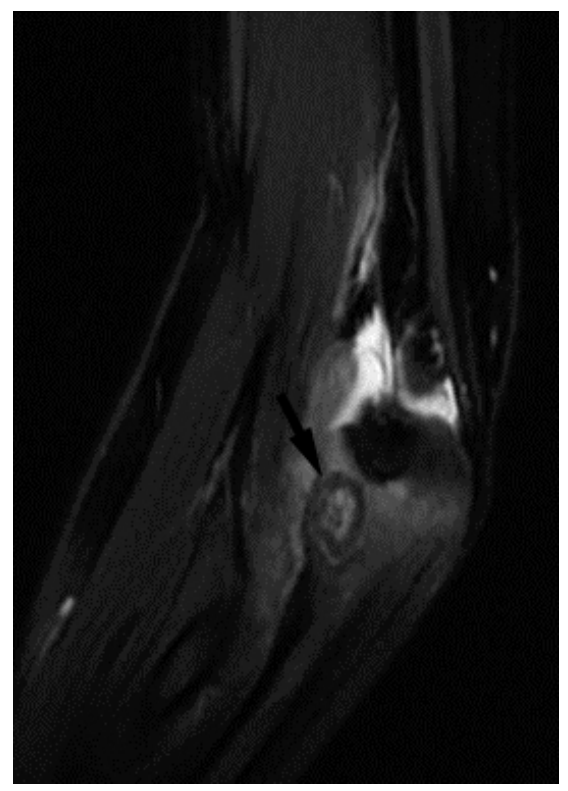

B

Figure 3. Coronal (A) and sagittal (B) magnetic resonance imaging of the left elbow showing the nidus at the coronoid process (arrow) with mild synovitis.

Various location have been identified, including the proximal ulna, the coronoid process of the ulna, the capitellum and the distal humerus [5]. In our case, the tumor was localized at the coronoid process of the ulna.

The most frequent symptom is persistent pain alleviated by NSAIDs, especially salicylate $[1,7,8]$. This may be due to the production of E2 prostaglandin, which is suppressed by anti-inflammatory medications $[2,7]$.

Signs of monoarthritis, including swelling and stiffness, are atypical for osteoid osteoma. The presence of synovitis may be confused with other more common etiologies of monoarthritis such us rheumatoid arthritis, neoplastic etiology, tuberculous arthritis, and osteochondritis dissecans. Such unspecific symptoms led usually to a delay in the diagnosis $[8,9]$.

The literature reported an average delay of 15 to 24 months from the first medical examination to the correct diagnosis [1].

Radiological features of osteoid osteoma include the nidus surrounded by reactive sclerosis, a joint effusion and periosteal reaction [10].

When CT scan is considered the modality of choice to define the actual nidus and surrounding cortical sclerosis, the simple X-ray is usually not helpful [5].

MRI is considered inferior to CT scan in detecting the nidus. The signal in the nidus is isointense to that of the muscle on T1-weighted images and is variable on T2-weighted images. However, this description is nonspecific [6].

MRI can be useful to demonstrate coexistent synovial proliferation or joint effusion and to exclude other comorbidities [6, 10].

Even with these examinations, identification of osteoid osteoma remains difficult since radiological abnormalities are not specific and need a high level of suspicion to be detected [1].

Conservative treatment which consists in pharmaceutical intervention (NSAIDs) can induce permanent relief of symptoms and regression of the nidus. If symptoms persist, definitive treatment with total excision of the nidus either by open surgery (wide resection or curettage) or by percutaneous treatment is required $[5,9]$.

\section{Ethical Statement \& Informed Consent}

Written informed consent was obtained from the patient's parents.

\section{Conflict of Interest}

The authors declare no conflicts of interest.

\section{Financial Disclosure}

This research received no specific grant from any funding agency in the public, commercial, or not-for-profit sectors.

\section{References}

[1] Ditsios K, Papadakis N, Theodoroudis I, Kostretzis L, Konstantinou P, Pinto I, et al. A misguiding osteoid osteoma in the bicipital tuberosity of the radius. Case Reports in Orthopedics. 2016;2016:1-3. Available from: https://doi.org/10.1155/2016/6428137

[2] Alrassasi YM, Almustafa MA, Al Eid ZM, Albattat MY, Al Batran KI. A rare case of intra-articluar osteoid osteoma of the elbow managed with arthroscopic excision. Cureus. 2021;13(6):e15666. Available from: https://doi.org/10.7759/cureus.15666 
[3] Savvidou OD, Koutsouradis P, Chloros GD, Papanastasiou I, Sarlikiotis T, Kaspiris A, et al. Bone tumours around the elbow: a rare entity. EFORT Open Reviews. 2019;4(4):133-142. Available from: https://doi.org/10.1302/2058-5241.4.180086

[4] P MJ, Kandathil JC, Jalal MJA, Manuel E, Theruvil B. Osteoid osteoma masquerading monoarthritis of the elbow. Clinical Rheumatology. 2021:131. Available from: https://doi.org/10.1007/s10067-021-05883-5

[5] Ge SM, Marwan Y, Abduljabbar FH, Morelli M, Turcotte RE. Arthroscopic management of intraand juxta-articular osteoid osteoma of the upper extremity: a systematic review of the literature. European Journal of Orthopaedic Surgery \& Traumatology. 2020;30(8):1333-1344. Available from: https://doi.org/10.1007/s00590-020-02710-6

[6] Akpinar S, Circi E. Arthroscopic excision of an intraarticular osteoid osteoma in the elbow joint. BMJ Case Rep. 2017;2017:bcr2017220868. Available from: https://doi.org/10.1136/bcr-2017-220868

[7] Bhatia DN. Arthroscopic excision of osteoid osteoma of the elbow. Arthroscopy Techniques. 2017;6(3):e543-e548. Available from: https://doi.org/10.1016/j.eats.2016.11.014
[8] Özdemir B, Akpina S. Arthroscopic excision of intraarticular subperiosteal osteoid osteoma of talar neck: a case report. Jt Dis Relat Surg. 2020;31(3):639-643. Available from: https://doir.org/10.5606/ehc.2020.71830

[9] Yano K, Kaneshiro Y, Sakanaka H. Arthroscopic excision for intra-articular osteoid osteoma of the olecranon fossa: a case report and literature review. Case Reports in Orthopedics. 2020;2020:1-6. Available from: https://doi.org/10.1155/2020/4034989

[10] Carneiro BC, Da Cruz IAN, Ormond Filho AG, Silva IP, Guimarães JB, Silva FD, et al. Osteoid osteoma: the great mimicker. Insights into Imaging. 2021;12(1):32. Available from: https://doi.org/10.1186/s13244-021-00978-8

Received: $2021-09-16$

Revision Requested: 2021-10-12

Revision Received: 2021-10-15

Accepted: $2021-10-18$ 